\title{
Utilidad del botón septal y nivel de satisfacción obtenido en los pacientes con perforaciones del septum: Nuestra experiencia
}

\section{Septal button utility and level of satisfaction obtained in patients with drilling septal. Our experience}

Raquel Artal S $^{1}$, Angel Urpegui G'1, José Ignacio Alfonso C ${ }^{1}$, Hector Vallés Varela.

\section{RESUMEN}

Introducción: La mayoría de las perforaciones septales suelen ser asintomáticas, aunque algunas pueden presentar una rinitis costrosa, obstrucción nasal, epistaxis recidivante o sibilancias nasales. Su origen es sobre todo iatrogénico o idiopático, pero también podemos encontrar otras causas. Los botones septales constituyen una solución útil por la sencillez de su colocación, siendo de elección en pacientes que no deseen una intervención quirúrgica más compleja.

Objetivo: Conocer las características de las perforaciones septales, además de, realizar un estudio sobre los resultados obtenidos en la cirugía de botón septal y el nivel de satisfacción obtenido por los pacientes tras esta intervención.

Material y método: Análisis retrospectivo de 22 pacientes intervenidos quirúrgicamente de colocación de botón septal entre enero de 2008 a agosto 2010 en el Hospital Clínico Universitario Lozano Blesa de Zaragoza.

El análisis incluye un estudio sobre la etiología, tamaño y sintomatología de dichas perforaciones candidatas a la utilización del botón septal. Además del análisis de la presencia o ausencia de complicaciones tras la cirugía, la evolución y la mejoría o empeoramiento posoperatorio, en cuanto a los cinco síntomas principales; obstrucción nasal, las sibilancias, las costras endonasales con sequedad, la epistaxis y la rinorrea con tres posibles respuestas; igual, mejor o peor. En el consultorio y a los tres meses de la intervención, se realiza una encuesta con una escala analógica visual (EVA), en la que el paciente realiza una puntuación subjetiva del 1 al 10 para valorar su nivel de satisfacción tras la colocación del obturador. Por último se le pregunta si volvería a ponérselo. Los resultados no fueron analizados con pruebas estadísticas dado el pequeño tamaño muestral.

Resultados: Nuestros pacientes han presentado una importante mejoría en síntomas como la obstrucción nasal y las sibilancias. La sequedad nasal ha sido la manifestación clínica con peores resultados tras la colocacion del botón. A nivel subjetivo el $77,2 \%$ de los pacientes se lo volverían a poner.

Conclusiones: Los botones septales constituyen un arma terapéutica más; a tener en cuenta para el cierre de las perforaciones septales con buenos resultados, en aquellos pacientes poco aptos para la correción quirúrgica.

Palabras clave: Perforación tabique nasal, botones septales, obturadores septales.

${ }^{1}$ Médico Otorrinolaringólogo. Hospital Clínico Universitario Lozano Blesa. Zaragoza, España 


\section{ABSTRACT}

Introduction: Most of the septal perforations are usually asymptomatic, although some may have a crusty rhinitis, nasal obstruction, recurrent epistaxis or nasal wheeze. Its origin is mostly iatrogenic or idiopathic but can also find other causes. Septal buttons are a useful solution for the simplicity of their placement, being of choice in patients who do not want surgery more complex.

Aim: Knowing the characteristics of septal perforations, in addition, a study on the results of septal button surgery and the level of satisfaction obtained by patients after the intervention.

Material and method: Retrospective analysis of 22 patients with surgical septal button surgical placement between January 2008 to August 2010 at the Hospital Clinico Universitario Lozano Blesa from Zaragoza.

The analysis includes a study on the etiology, symptoms of such size and drilling candidates for the use of septal button. Besides the analysis of the presence or absence of complications after surgery, and postoperative improvement or worseningin the five main symptoms, stuffy, nose, wheezing, endonasal crust with dryness, epistaxis and rhinorrhea with three possible answers, same, better or worse. Three months after surgery in the office is conducting a survey with a visual analogue scale (VAS), in which the patient makes a subjective score of 1 to 10 to assess their level of satisfaction after the placement of the shutter. Finally asked if he would wear it. The results were analyzed with statistical tests given the small sample size.

Results: Our patients have experienced a significant improvement in symptoms such as nasal obstruction and wheezing. Nasal dryness has been with worse clinical outcomes after placement of the button. Subjectively $77.2 \%$ of patients would put him back.

Conclusions: The septal buttons are a therapeutic tool to keep in mind for the closure of septal perforations with good results in patients unsuitable for surgical correction.

Key words: Nasal septum perforation, septal buttons, plugs, septal.

\section{INTRODUCCIÓN}

Las perforaciones septales suelen ser en la mayoría de los casos asintomáticas. En aquellas situaciones en las que aparece sintomatología es cuando el paciente acude al especialista.

La perforación del septum nasal afecta el funcionamiento de la nariz, al alterarse el flujo laminar, lo que provoca cambios en la corriente de aire inspirado con disminución de la velocidad global del flujo aére $0^{1-3}$.

Al manifestarse, pueden presentar varios síntomas nasales. Los cinco síntomas más frecuentes que el paciente refiere son la obstrucción nasal, las sibilancias, las costras endonasales con sequedad, la epistaxis y la rinorrea. Pero también puede aparecer en menor frecuencia dolor, hiposmia, rinolalia, infecciones e incluso cacosmia.
La etiología puede variar y se agrupa en diversos grupos:

- Traumáticas/iatrogénicas. Septoplastías y septorrinoplastías, el rascado nasal, hematomas de tabique postraumáticos, pericondritis del tabique, cirugía por poliposis nasosinusal, cauterización bilateral simultánea, taponamientos bilaterales mediante sondas con balón y sondas nasogástricas o nasotraqueales.

- Tóxicas. Principalmente la cocaína pero también otros irritantes inhalados como ácido crómico, ácido sulfúrico, carbonato de sodio, nitrato de calcio, arsénico, mercurio y fósforo.

- Productos tópicos nasales. Vasoconstrictores y en menor medida corticoides locales ${ }^{2,3}$.

- Infecciosas. Bacterianas: sífilis, difteria, leishmania y fiebre tifoidea, o micóticas: aspergilo- 
sis, histoplasmosis, criptococosis y actinomicosis.

- Neoplásicas. Carcinomas, leucemias e incluso granulomas de la línea media.

- Vasculitis. Como la enfermedad de Wegener, el lupus y la sarcoidosis y por último;

- Las perforaciones idiopáticas, entre ellas la úlcera de Hajek.

Las causas más frecuentes son las de origen iatrogénico, seguidas de las idiopáticas.

Según la localización, las perforaciones anteriores son las predominantes y suelen manifestarse con sibilancias, mientras que las posteriores suelen dar menos clínica por la humedad que aportan los cornetes.

Una perforación que no produce síntomas, en un principio no tiene indicación de tratamiento quirúrgico. Como medidas conservadoras utilizamos nebulizaciones 0 irrigadores que proporcionan humedad, ya sea con solución salina u otras soluciones que tarden mayor tiempo en vaporizarse, emolientes (vaselina y/o glicerina) 0 pomadas con tratamiento antimicrobiano.

Las indicaciones para reparar quirúrgicamente las perforaciones, incluyen a los pacientes sintomáticos en los que han fallado los métodos conservadores. Se han diseñado múltiples técnicas para el cierre de las perforaciones; la mayoría se basan en desplazamientos de colgajos de mucosa nasal o labial, y casi todas útiles para perforaciones de tamaño pequeño, mediano y grande. Si bien, son las perforaciones pequeñas las que más posibilidades tienen de cierre quirúrgico.

Uno de los capítulos menos satisfactorios en la cirugía nasal es el cierre de las perforaciones mayores de $1 \mathrm{~cm}$. Para estas perforaciones, el botón septal es un método alternativo al de la cirugía, poco agresivo para el paciente y de colocación fácil y rápida. En la actualidad se utilizan dos tipos de materiales para elaborar estas prótesis: el elastómero de silicona y las resinas acrílicas. Están contraindicados en infecciones del tabique con osteítis, en enfermedades crónicas del tabique (Wegener) y en neoplasias o perforaciones extremadamente largas y grandes. No se recomienda en los casos de deformidades septales, dado que el disco del lado convexo de la deformidad podría aumentar la obstrucción nasal.
Nuestro objetivo es realizar un estudio sobre dicha patología, teniendo en cuenta las causas, el tamaño y los principales síntomas que conlleva la perforación septal. Valoramos posteriormente, el nivel de satisfacción obtenido por los pacientes en los que se ha utilizado el obturador septal para el cierre.

Sus resultados son buenos, con unas tasas de éxito alentadoras en la literatura. Constituye un arma terapéutica a tener en cuenta por parte del especialista.

\section{MATERIAL Y MÉTODO}

Presentamos un estudio de análisis retrospectivo de la casuística obtenida de 22 pacientes que fueron intervenidos quirúrgicamente de colocación de botón septal entre enero de 2008 a agosto de 2010, en nuestro hospital. Todos ellos presentaban sintomatología que no cedía con medidas conservadoras y un tamaño de perforación asequible para la utilización del obturador. En todos los pacientes hemos usado el mismo método para cerrar la perforación, un obturador (botón septal) formado por dos discos, unidos por un eje central. El disco tiene un diámetro de 3,2 cm y está elaborado con silicona (Figura 1). Estos botones han sido fabricados en Florida (USA), por la compañía Invotec International.

El análisis de los 22 pacientes con botón septal incluye: un estudio de la etiología presentada, los tamaños de las perforaciones de la muestra, la presencia 0 ausencia de complicaciones tras la cirugía, la evolución posoperatoria y la mejoría 0 empeoramiento en cuanto a los cinco síntomas principales; obstrucción nasal, las sibilancias, las costras endonasales con sequedad, la epistaxis y la rinorrea con tres posibles respuestas; igual, mejor 0 peor. Posteriormente en el consultorio se realiza una encuesta con una escala analógica visual (EVA), en la que el paciente realiza una puntuación subjetiva del 1 al 10 para valorar su nivel de satisfacción tras la colocación del obturador. Por último se le pregunta si volvería a ponérselo. Mostramos resultados descriptivos. Éstos no fueron analizados mediante una estadística inferencial, debido al pequeño tamaño muestral del que disponemos para obtener resultados estadísticamente significativos. 


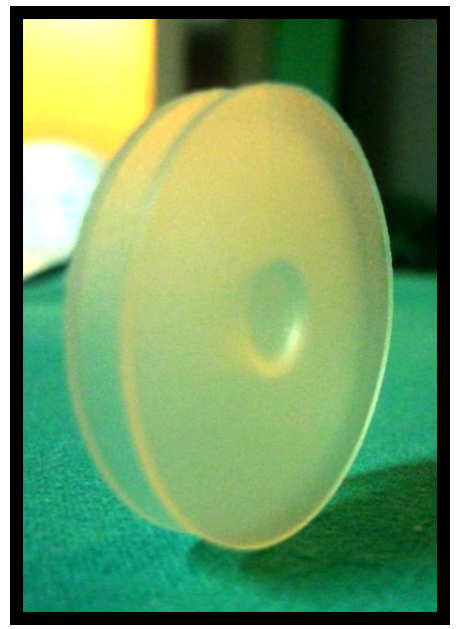

Figura 1. Botón septal utilizado en el cierre de las perforaciones del septum.

\section{RESULTADOS}

La edad media de la población a estudio es de 45,9 años, con un rango de edad de 34 a 60 años. En la distribución por sexos predomina el sexo masculino $(59,1 \%)$ sobre el femenino (40,9\%).

Las principales causas de perforación septal que nos hemos encontrado por orden de frecuencia han sido: idiopática, iatrogénica, traumatismos, cocaína y abuso de nebulizadores (Figura 2). Dentro de las causas iatrogénicas, encontramos un ligero predominio de septorrinoplastías (13,6\%) frente a septoplasias $(9,1 \%)$. Debido a que en nuestro estudio hemos excluido todas aquellas perforaciones de septum asintomáticas (al no ser considerada indicación de cirugía), la causa iatrogénica, que en numerosos estudios es la más frecuente, aquí pasa a un segundo lugar.

Las consecuencias aerodinámicas de una perforación septal son frecuentes. Los síntomas que presentan los pacientes tienen relación con el tamaño y la localización de la perforación. En nuestra muestra los dos síntomas principales que manifiestan los pacientes con perforación septal fueron la obstrucción nasal y la sequedad asociada a costras. Las sibilancias también han aparecido en un importante porcentaje seguidas por episodios de epistaxis y rinorrea (Tabla 1 ).

Un total de $16(72,72 \%)$ de las 22 perforaciones en las que hemos utilizado esta técnica son de

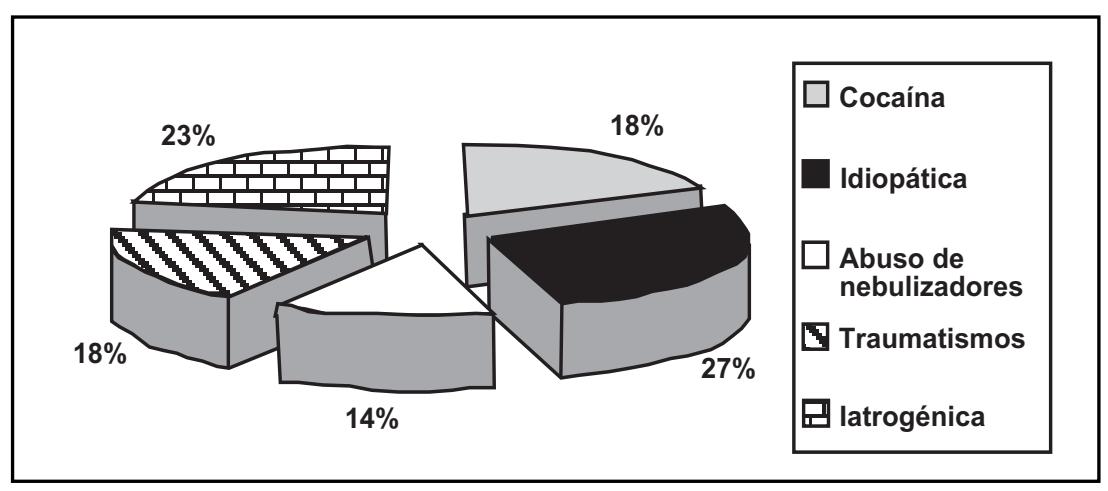

Figura 2. Causas de las perforaciones septales en nuestra muestra. 
Tabla 1. Sintomatología principal de las perforaciones septales de la muestra

\begin{tabular}{|lcc|}
\hline Síntomas & Pacientes afectados & $\%$ \\
\hline Obstrucción nasal & 22 & 100 \\
Sibilancias & 19 & 86,4 \\
Sequedad nasal/costras & 22 & 100 \\
Epistaxis & 12 & 54,6 \\
Rinorrea & 11 & 50 \\
\hline
\end{tabular}

un tamaño mediano $(1 \mathrm{~cm}-2 \mathrm{~cm})$. El resto han sido en perforaciones grandes y aisladamente una pequeña de tamaño próximo a $1 \mathrm{~cm}$ (Tabla 2).

En el $55 \%$ (13) de los pacientes, se les colocó únicamente el botón septal. En 35\% (7) además del botón, se realizó una turbinoplastía con radiofrecuencia. Otro paciente $(4,5 \%)$ además del botón y de la turbinoplastía, requirió cirugía endoscópica por poliposis nasosinusal y sinusitis. Y un último paciente $(4,5 \%)$ con estenosis valvular bilateral fue sometido a valvuloplastía en el mismo acto quirúrgico.

La colocación aislada del botón septal la hemos realizado mediante una cirugía ambulatoria con anestesia local tópica. En aquellas situaciones en las que realizábamos un procedimiento quirúrgico adicional, además de la colocación del botón, la anestesia requerida fue general.
Tabla 2. Tamaño de las perforaciones septales de nuestra población a estudio

\begin{tabular}{|lcc|}
\hline Tamaño & Pacientes afectados & $\%$ \\
\hline $0-1 \mathrm{~cm}$ & 1 & 4,5 \\
$1 \mathrm{~cm}-2 \mathrm{~cm}$ & 16 & 72,72 \\
$>2 \mathrm{~cm}$ & 5 & 22,72 \\
Total & 22 & 100 \\
\hline
\end{tabular}

En todos los casos hemos seguido la misma sistemática; se evalúan las dimensiones de la perforación mediante la introducción de una lámina de Espongostan ${ }^{\circledR}$ por una narina que cubra la perforación, y por la otra, un hisopo tintado con azul de toluidina. Según el tamaño de la perforación, recortamos el botón, dejando unos milímetros de márgenes sobrantes. El disco siempre ha de ser más ancho que la perforación para que cubra sus bordes. Una TC también puede ser útil y permite apreciar el tamaño de la pérdida de sustancia con un margen de error de 1-2 $\mathrm{mm}^{4}$.

A continuación preparamos la prótesis para introducirla. En uno de los discos damos un punto de sutura juntando los márgenes anterior y posterior. $Y$ un segundo punto, juntando los márgenes superior e inferior (Figura 3).

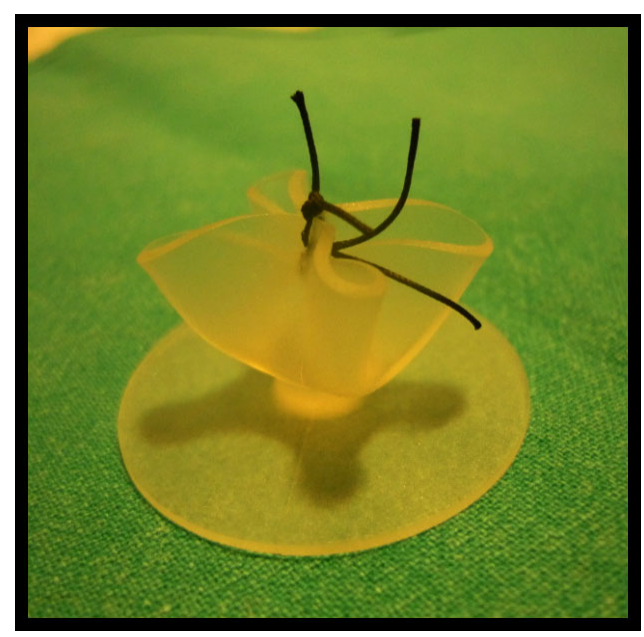

Figura 3. Punto en uno de los discos del botón para favorecer la introducción a través de la perforación. 
Para la colocación del botón, introducimos por una narina la prótesis y con una pinza hacemos pasar el disco plegado a la otra fosa a través de la perforación. Por la otra fosa traccionamos y seccionamos los puntos de manera que el disco se repliega y se coloca cubriendo los márgenes. Para ajustarla basta con hacerla girar (Figura 4).

Hay que tener mucho cuidado para que los discos del botón no toquen la unión del septum con el cartílago lateral superior, dado que esto sería una sensación particularmente desagradable para el paciente.

Los primeros días tras la cirugía, recomendamos a los pacientes lavados con suero fisiológico frecuentes, además de una solución oleosa que contenga antibiótico y/o antiinflamatorio con el objetivo de evitar la aparición de costras en la superficie del botón septal, que ocasionarían la obstrucción. Se les aconseja no sonarse la nariz de forma brusca. Todos los pacientes son seguidos posteriormente en nuestras consultas externas.

Hemos llevado a cabo una encuesta a los 22 pacientes con botón septal. La mayoría de ellos (13 pacientes) con cirugía sólo de botón y en 9 pacientes se realizó un procedimiento quirúrgico adicional el cual, parece influir en los resultados.

Nuestros resultados deben de tomarse con cierta precaución ya que son datos descriptivos preliminares. Serían necesarios estudios similares sobre una muestra más amplia, en la que podamos comparar el nivel de satisfación obtenido en dos grupos de igual tamaño, y con otros mecanismos de control para evitar sesgar los resultados.

Si bien existe una pequeña diferencia en cuanto al nivel de satisfacción obtenido entre los casos de uno y otro grupo, dado el bajo número de población en cada muestra el estudio no es significativo para que tenga un valor concluyente.

Se ha evaluado la presencia 0 ausencia de complicaciones tras la cirugía, la evolución posoperatoria y la mejoría o empeoramiento en cuanto a los cinco síntomas principales; obstrucción nasal, las sibilancias, las costras endonasales con sequedad, la epistaxis y la rinorrea con tres posibles respuestas; igual, mejor 0 peor. La encuesta termina con una escala analógica visual (EVA), en la que el paciente realiza una puntuación subjetiva del 1 al 10 para valorar su nivel de satisfacción tras la colocación del obturador. Por último se le pregunta si volvería a ponérselo.

La encuesta se ha realizado en las consultas de nuestro hospital. Se les decía expresamente que su participación era voluntaria y que la información recopilada tendría un tratamiento meramente estadístico.

La principal complicación que nos hemos encontrado ha sido la mala tolerancia del paciente ante el botón. En cuatro pacientes (18,2\%), han presentado molestias y dolor los primeros días tras la intervención, disminuyendo progresivamente. Un paciente $(4,5 \%)$, presentó estornudos frecuentes en las primeras horas del posoperatorio. Seis

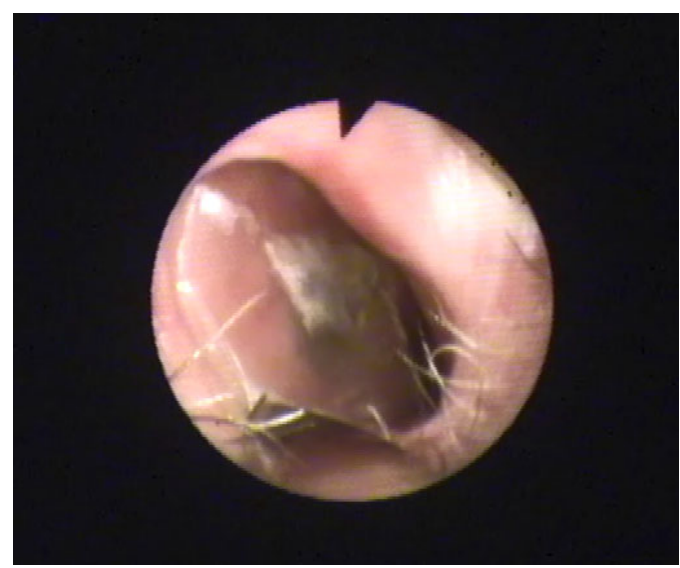

Figura 4. Botón colocado. Visión por fosa nasal izquierda. 
de los pacientes $(27,2 \%)$ afirmaban continuar con sequedad nasal. Y en cinco (22,7\%), aparecieron erosiones en la mucosa, por el roce y el decúbito del obturador en el suelo de la fosa. En estas lesiones por decúbito, en las consultas y con el paciente despierto, fue necesario recortar la parte inferior del disco que estaba provocando la lesión.

Sólo un paciente $(4,5 \%)$ presentó varios episodios de infección y sinusitis, lo que provocó la manipulación del botón por parte del paciente, con la consiguiente extrusión.

Otro de ellos $(4,5 \%)$, refirió que al sonarse la nariz el botón se movió y posteriormente él mismo lo extrajo.

Epistaxis leves aparecieron en tres pacientes $(13,6 \%)$ las primeras $24-48$ horas tras la colocación, cediendo paulatinamente con las medidas higiénicas pautadas (lavados y pomada) en dos. El último requirió la extracción del botón por parte del cirujano ya que presentaba episodios de sangrado frecuentes y autolimitados, pero muy molestos para el paciente.

De los 22 pacientes, un total de 19 (86,3\%) mantienen su botón actualmente, mientras que en 3 pacientes (13,7\%) fue necesaria la retirada 0 se produjo la extrusión.

Al analizar los cambios que el paciente experimenta en la sintomatología nasal tras la colocación del botón, lo más llamativo es la importante mejoría en la obstrucción nasal (100\%) y en las sibilancias. En todos los pacientes que se habían presentado sibilancias (19), éstas desaparecieron al terminar con un flujo aéreo turbulento anárquico, recuperando el flujo laminar fisiológico.

La sequedad nasal se presentó en los 22 pacientes, y sólo el 59\% comentan una clara mejoría.

La epistaxis se presentó en 12 pacientes y 10 de ellos afirmaban estar mucho mejor, sin presentar ningún episodio de sangrado posterior.

La rinorrea mejoró en 8 pacientes de los 11 que la presentaban (Figura 5).

Los peores resultados aparecen al valorar las costras y la sequedad. Ocho de los pacientes dicen estar igual y uno peor.

A los tres meses de la cirugía, se les preguntaba en la consulta, cuál era su nivel de satisfacción en una escala (escala analógica visual, EVA) del 1 al 10 (máximo nivel de satisfacción), la media resultante de todas las respuestas obtenidas ha sido de 7,1. [Max 10, Min 3, desviación típica $1,58]$.

Si separamos los datos obtenidos en la encuesta, sobre el nivel de satisfacción según los grupos quirúrgicos: los pacientes que han sido sometidos a una cirugía única y exclusiva de botón

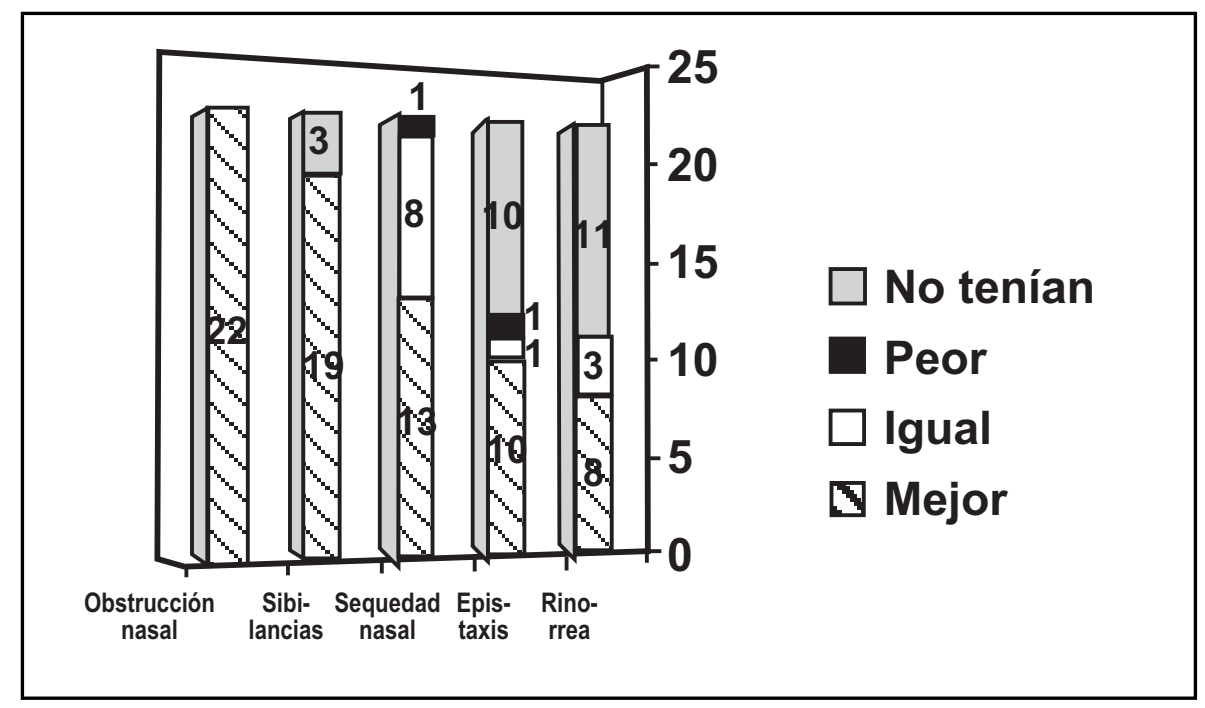

Figura 5. Evolución de la sintomatología posoperatoria tras la colocación del botón septal. 
septal, presentan una puntuación media en la escala EVA de 6,56 [Max 8, Min 4,5, desviación típica 1,16], mientras que los sometidos a cirugía de botón septal conjuntamente con otros procedimientos quirúrgicos (turbinoplastía, valvuloplastía y cirugía endoscópica), presentaban una puntuación media de 7,61 [Max 10, Min 3, desviación típica 1,94], cifra ligeramente superior. Por tanto, podemos concluir que la mejoría con el botón septal es muy satisfactoria, siendo todavía superior, si añadimos otros procedimientos quirúrgicos que ayudan a mejorar el bienestar del paciente.

Dada la subjetividad de las respuestas y al apreciar que nos encontrábamos con pacientes disconformes e insatisfechos, posteriormente, para hacer una valoración global, se les preguntaba al grupo completo si se lo volverían a poner. Resulta llamativo que el $77,2 \%$ (17) sí que lo harían. El $18,2 \%$ (4) tal vez lo harían y sólo 4,5\% (1), no lo haría.

\section{DISCUSIÓN}

Diferentes técnicas han sido utilizadas para el cierre de las perforaciones septales. Uno de los primeros intentos realizados a principios del siglo pasado consistía en ampliar la perforación, esperando que las molestias disminuyesen. En los años 40 se describieron los primeros colgajos de transposición septal utilizando injertos de tejido conectivo a modo de sandwich entre la mucosa septal. Cottle fue el primero en descubrir el avance del colgajo bipediculado para el cierre de perforaciones septales. Varias técnicas usando colgajos de cornete inferior y medio fueron descritas a mediados de los 50 y principios de los 60 . Tripton describe el colgajo labio-bucal en 1970 y Tardy introduce el colgajo mucoso sublabial en 1977. También se han utilizado autoinjertos de fascia de temporal y mucopericondrio de concha. Y más recientemente periostio mastoideo, restos óseos y cartilaginosos del propio tabique, vómer, lámina perpendicular del etmoides y cresta ilíaca ${ }^{3,5-10}$.

Los botones septales fueron descritos inicialmente por Meyer y Link en 1951311.

Inicialmente se elaboraban a mano, pero a partir de 1975, se preparaban obturadores estándar, dirigidos a perforaciones de $0,5 \mathrm{a} 3 \mathrm{~cm}$.
En la actualidad se utilizan dos materiales para elaborar estas prótesis: el elastómero de silicona y las resinas acrílicas de introducción más reciente.

Las principales causas de perforación septal que hemos encontrado en nuestra muestra han sido en primer lugar la idiopática y posteriormente la iatrogénica. Según la literatura, ambas son la etiología más frecuente, salvo que la iatrogénica precede a la idiopática. Nuestro porcentaje de causas iatrogénicas es igual o ligeramente inferior al de otros autores $2,12,13,15,17$. Debemos comentar que en nuestro hospital tenemos formación para el médico interno residente, lo que dada la menor experiencia y el periodo de aprendizaje, puede contribuir a aumentar estos datos.

Es de destacar el aumento de perforaciones en la bibliografía revisada a consecuencia de los descongestionantes nasales (vasoconstrictores) y en menor medida esteriodes nasales ${ }^{2,10}$. Se ha de informar a los pacientes que su uso abusivo puede acarrear sangrado y perforación septal.

Al igual que todos los autores que hemos revisado, las complicaciones más importantes que han aparecido, infecciones, epistaxis y extrusión, derivan de la mala tolerancia a la prótesis.

Luff et a ${ }^{12}$ presentan una serie de resultados en la que sólo el $45 \%$ de los pacientes mantienen su botón en un periodo de seguimiento acumulado de 10 años. Mullace ${ }^{13}$ y Eliachar ${ }^{14,15}$ presentan $70 \%$ de pacientes que permanecen con el obturador in situ. De nuestros 22 pacientes, 86,3\% todavía presentan su botón septal ocluyendo la perforación, aunque nuestro estudio se realiza en un corto periodo de tiempo por lo que no se puede comparar con otros trabajos. Los tres casos en lo que se ha extruido o ha necesitado la extracción se han presentado en los primeros tres meses.

La principal mejoría clínica observada tras realizar la encuesta ha sido a nivel de la obstrucción nasal y las sibilancias. Mientras que aproximadamente $40 \%$ de los pacientes se encuentran igual 0 peor en cuanto a sequedad y costras. Estos datos concuerdan con lo aportados por Osma et $\mathrm{al}^{16}$. Se precisa de cuidados minuciosos locales para evitar la aparición de costras nasales.

Federspil et al ${ }^{17}$ presentan una serie de 57 pacientes a lo largo de 7 años con botón septal que lo mantienen el $75 \%$ de ellos. En la escala analógica visual (EVA) presentan un nivel de satis- 
facción de 8,3. Nuestros pacientes presentan un nivel de satisfacción ligeramente más bajo 7,1 , aunque el $77,2 \%$ de ellos se lo volverían a poner, lo que nos hace suponer que infravaloran su nivel de satisfacción al puntuar en la escala.

Nuestros resultados deben de tomarse con cierta precaución ya que son datos descriptivos preliminares. Al separar los dos grupos quirúrgicos (cirugía única de botón septal y cirugía de botón septal junto con otro procedimiento quirúrgico) el nivel de satisfacción en la EVA pasa de 6,5 en el primer grupo a 7,6 en el segundo. Serían necesarios estudios similares sobre una muestra más amplia, en la que podamos comparar el nivel de satisfacción obtenido en dos grupos de igual tamaño, y con otros mecanismos de control para evitar sesgar los resultados.

Los obturadores septales nos parecen una técnica útil, con resultados satisfactorios y alentadores ${ }^{18,19}$. Son de fácil manejo y cuentan con la ventaja de su uso ambulatorio. Es el tratamiento de elección en las perforaciones sintomáticas de pacientes frágiles 0 que no desean someterse a intervenciones quirúrgicas de mayor envergadura.

\section{BIBLIOGRAFÍA}

1. Re M, Paolucci L, Romeo R, Mallardi V. Surgical treatment of nasal perforations. Our experience. Acta Otorrinolaryngol Ital 2006; 26(2); 1029.

2. Dosen LK, Haye R. Nasal septal perforation 1981-2005. Changes in etiology, gender and size. Ear Nose Throath Disord 2007; 7: 7-11.

3. Thomassin JM, Forma C, Danvin JB, Bailhache A. Cirugía de las perforaciones septales. Elsevier Masson, 2009: 1-17.

4. Price DL, Sherris DA, Kern EB. Computed tomography for constructing custom nasal septal buttons. Arch Otolaryngol Head Neck Surg 2003; 129: 1236-123.

5. Palomar V, Cervera Paz FJ. Surgical technique for the reconstruction of the nasal septum: the pericranial flap. Head Neck 2000; 22: 90-4.
6. Ribeiro JS, Silva DA, Silva G. Technical advances in the correction of septal perforation associated with closed rhinoplasty. Arch Facial Plast Surg 2007; 9: 321-7.

7. Woolford TJ, Jones NS. Repair of nasal septal perforations using local mucosal flaps and a composite cartilage graft. J Laryngol Otol 2001; 115: 22-5.

8. Kyung Chul L, No Hee L, Jae Ho B, Sung Min Jin. Surgical treatment using an allograft dermal matrix for nasal septal perforation. Yonsei Med J 2008; 49(2): 244-8.

9. López lizarraga E, López Demeritus E, Bañuelos R, Saavedra Martinez Jl, Troyo San Román R, Macías Beltrán JM. Modalidad en el cierre de la perforación septal anterior con transposición de la lámina perpendicular del etmoides. An Orl Mex 2009; 54(1): 1-6.

10. KRIDEL RWH. Considerations in the etiology, treatment and repair of septal perforations. Facial Plast Surg Clin N Am 2004; 12: 43550.

11. Galindo CN, Ortega del Alamo P, Delgado-Urena MP. The septal button: an alternative to reparative surgery of septal perforations. Acta Otorrinolaringol Esp 1992; 43: 102-4.

12. Luff DA, Kam A, Bruce IA, Willatt DJ. Nasal septum buttons: symptom scores and satisfaction. J Laryngol Otol 2002; 116: 10014.

13. Mullace M, Gorinı E, Sbrocca M, Artesi L. Mevio $N$. Management of nasal septal perforation using silicone nasal septal button. Acta Othorhinolaryngol Ital 2006; 26(4): 216-8.

14. Eliachar I. Mastros NP. Improved nasal septal prothestic septal button. Otolaryngol 1995; 112: 347-9.

15. DøSen LK, HAYe R. Silicone button in nasal septal perforation. Long term observations. Rhinology 2008; 46(4): 324-7.

16. Osma U, Cüreoglu S, Akbulut N, Meriç F, Topçu I. The results of septal button insertion in the management of nasal septal perforation. $J$ Laryngol Otol 1999; 113: 823-4.

17. Federspil PA, SchneIder M. The custom made septal button. Laryngorhinotologie 2006; 85(5): 323-5. 
18. Blind A, Hulterström A, Berggren D. Treatment of nasal septal perforations with a custommade prosthesis. Eur Arch Otorhinolaryngol 2009; 266: 65-9.
19. Barraclough JP, Ellis D, Proops DW. A new method of construction of obturadors for nasal septal perforations and evidence of outcomes. Clin Otolaryngol 2007; 32(1): 51-4.

Dirección: Raquel Artal Sánchez

C/ La lectura $n^{0} 133^{\circ} \mathrm{dch}$

50015, Zaragoza (España)

E mail: raquelartal@hotmail.com 